\title{
CLASSICAL AND CONNECTIONIST MODELS: LEVELS OF DESCRIPTION
}

ABSTRACT. To begin, I introduce an analysis of interlevel relations that allows us to offer an initial characterization of the debate about the way classical and connectionist models relate. Subsequently, I examine a compatibility thesis and a conditional claim on this issue.

With respect to the compatibility thesis, I argue that, even if classical and connectionist models are not necessarily incompatible, the emergence of the latter seems to undermine the best arguments for the Language of Thought Hypothesis, which is essential to the former.

I attack the conditional claim of connectionism to eliminativism, presented by Ramsey et al. (1990), by discrediting their discrete characterization of common-sense psychological explanations and pointing to the presence of a moderate holistic constraint.

Finally, I conclude that neither of the arguments considered excludes the possibility of viewing connectionist models as forming a part of a representational theory of cognition that dispenses with the Language of Thought Hypothesis.

In the debate about classical and connectionist models the way different levels of description relate constitutes a central issue. There is, however, considerable confusion concerning what is exactly meant by 'level of description' and the kinds of interlevel connections that may be relevant to the debate. I have, then, considered it appropriate to start off by characterizing in general terms the notion of 'description level' and the types of interlevel correlations that may be involved in the definition of the two models under consideration.

Subsequently, the particular terms of the debate are put forward. Thus, I indicate the description levels that a realist understanding of folk psychology will assume, and introduce classical and connectionist models as two different attempts to account for the sort of cognitive processing that such folk psychology would postulate. ${ }^{1}$ Of course, these two models differ in the nature of their account. For classical models define their fundamental description level as one whose states are constituted by syntactic formulae and computational relations to these syntactic formulae, so that they are essentially committed to the Language of Thought Hypothesis; ${ }^{2}$ connectionist models, on the other 
hand, will characterize their basic states as connectivity patterns and activation patterns over very simple units.

The question I want to address in this paper is whether connectionist models will be able to account for common-sense psychological states in spite of dispensing with the Language of Thought Hypothesis. For a positive answer to this question would constitute a major threat to classical models, at least as realist models of our cognitive capacities, since the fundamental argument for the Language of Thought Hypothesis has the structure of an inference to the best explanation. An argument of this nature only holds if no alternative, better grounded explanation is available. But what I plan to discuss is precisely whether there is any principled reason to rule out the idea that connectionist models can provide such an alternative account. This is why I envisage the main question examined in this paper as crucial to the connectionist debate.

More concretely, I will seek to rebut two lines of argumentation that can be construed as defending the indispensability of classical models and, therefore, rejecting the idea that connectionist models could furnish such an alternative account. The first argument appears in Fodor and Pylyshyn (1988). Their basic claim is that connectionist models are not at a cognitive level but at an implementation level, so that they can only account for common-sense psychological states insofar as they implement classical models. Consequently, a connectionist account of our cognitive processes can hardly aim to dispense with the Language of Thought Hypothesis.

The second argument, due to Ramsey, Stich, and Garon, ${ }^{3}$ attempts to ground a conditional claim that goes from connectionism to eliminativism on the basis that no systematic correlation can be established between common-sense psychological states and connectionist states, given that the former are, in their own terms, propositionally modular and the latter are holistically individuated. An inverted reading of this conditional claim will certainly lead to an argument that goes from folk psychology to a rejection of connectionism.

My rebuttal of Fodor and Pylyshyn's argument points to the idea that they misconceive the kind of task that a representational theory of cognition is supposed to do and the level at which it can define its most basic types of states. With respect to the second argument, I shall oppose to the idea that common-sense propositional attitudes are basically discrete, and vindicate an interpretation of folk psychology, 
which, despite granting a moderate holistic constraint, is still compatible with a certain degree of discreteness in the individuation of our propositional attitudes. I will also use the central elements of my former replies to elaborate a pair of arguments against the plausibility of classical models as a realist approach to our cognitive capacities.

To conclude, I should stress that, in this paper, I do not intend to argue that no principled reason can be supplied against the idea of elaborating, on the basis of connectionist models, a representational theory of cognition that dispenses with the Language of Thought Hypothesis. I only aim to show that neither of the two lines of argumentation under consideration provides such a principled reason.

\section{THE TERMS OF THE DEBATE}

Fixing the terms of the debate about connectionism requires previous understanding of what is meant by levels of description and the distinct ways one can expect them to relate. Hence, I devote Section 1.1 to clarifying the notion of 'description level' that I intend to employ in the discussion, and the kinds of interlevel correlations that, in my view, are implicated in the debate. Section 1.2 will provide a particularized presentation of the description levels that are involved in folk psychology, and in classical and connectionist models.

\subsection{Comparing Description Levels}

We can characterize a description level by the sort of state that it stipulates and assume that, in general, state types are defined by two components: a set of objects and a set of properties. ${ }^{4}$ Different description levels can surely be compared in virtue of the sor 2 of state that they postulate. In my view, two basic ways of carrying out such a comparison will be relevant to our debate.

(i) One way of comparing two description levels will be to examine the systematic correlations or mappings ${ }^{5}$ that can be established among the sets of properties that define their respective state types. A comparison of this kind is made when, for instance, we detect a systematic correlation between a macroscopic property, like 'being a liquid', and certain molecular properties. In general, the strength of the link between any two given sets of properties will certainly depend on the number and 
character of the systematic correlations that can be established among their respective properties.

On some occasions, however, even though two sets of properties may not hold any strict systematic correlation because they are defined with a different degree of vagueness, we may still be reluctant to deny the existence of any sort of systematic correlation. In fact, we may feel inclined to say in some cases that an approximate mapping can be established between them, where approximation is clearly a matter of degree. One can expect this situation, for instance, in comparing common-sense and scientific description levels and, consequently, it will surely be present in any attempt to account for folk psychology by means of either classical or connectionist models.

(ii) A different way of exploring the possible connections between description levels will be to compare the properties that we are prepared to ascribe to them from a metalevel of description that takes the states of lower description levels as objects of their own states.

An intentional realist like Fodor ${ }^{6}$ would contend, for instance, that, in folk psychology, there is a description level whose objects are propositional attitudes, individuated by their propositional content and their psychological mode. He would also add that propositional attitudes have constituent parts and are productive, systematic, and compositional. On my account, I should say, however, that these additional properties integrate a metalevel of description whose state types include common-sense propositional attitudes as the objects to which the aforementioned properties are ascribed. As we shall see, a central factor in the debate about classical and connectionist models consists in determining the sequence of metaproperties that can be truly applied to common-sense propositional attitudes, and whether connectionist states could share these metaproperties.

A chain or hierarchy of description levels can often be established on the basis of these two types of connections. The explanatory power of a chain will certainly be a function of the strength and character of such connections. Moreover, I should say that two chains of description levels can be compared through the analysis of the correlations that can be established among their component description levels. The interest of the comparison will be a function of the strength of the correlation 
and the place that the description levels under consideration occupy in their respective chains.

It is difficult, however, to supply general criteria for deciding what makes a set of description levels a chain, when two description levels belong to the same chain, or the number of description levels a chain consists of. In fact, the way in which one decides to group and differentiate description levels will partly depend, in my opinion, on specific pragmatic interests. Thus, the characterization I will set forth in the next subsection will be explicitly mediated by my interest in sorting out some aspects of the current debate about connectionism and, consequently, it will miss distinctions that might be essential to a different approach.

Let us now use the distinctions introduced in this subsection to set the terms of the debate about classical and connectionist models.

\subsection{Description Levels in Classical and Connectionist Models for Folk Psychology}

We can assume, for now, that both models coincide in seeking to show the scientific respectability of the basic ontological commitments and generalizations of folk psychology. Thus, intentional realists present folk psychology as a theory about the mind that is committed to the existence of mental states that are causally connected precisely in virtue of their content. The structure of such mental contents coincides with that of the sentences that are used to ascribe them.

One of the structural properties of these sentences, and therefore of our mental contents, is that they have constituent parts, ${ }^{7}$ namely, parts that in construction with others supply a molecular content. Related properties of contentful mental states are their productivity, systematicity, and compositionality. The notion of productivity is linked to what Chomsky calls the generative character of linguistic competence, namely, the possibility of generating or understanding an unbounded number of sentences. By systematicity, we understand the fact that the production or understanding of certain mental contents is intrinsically connected to the production or understanding of some others. Finally, compositionality refers to the stability of the semantic contribution of each constituent part across different molecular representations in which they occur.

This is the characterization of folk psychology that classical models 
propose as a starting point in their account of cognitive capacities. If we apply our conceptual apparatus to this common-sense psychological theory, we can say that it defines a chain composed of two description levels. ${ }^{8}$

Description level Folk.1: Propositional attitudes are the states of this description level. They are individuated by their propositional content and their psychological mode, which, in turn, determine their causal powers. For example, we could say, at this description level, that a subject $\mathrm{S}$ has the belief that $\mathrm{aRb}$ at time $\mathrm{t}$, where $(\mathrm{S}, \mathrm{a}, \mathrm{b})$ will be the objects that compose the psychological state; whereas the relational property ' $R$ ', the psychological belief mode, and certain causal powers will count among the properties that can be truly ascribed to these objects at this description level.

Description level Folk. $2:^{9}$ Propositional attitudes appear as the objects of the states posited by this description level. We are, then, dealing with a metalevel of description. Some of the properties that are ascribed to Folk.1 states from this metalevel refer, as we have seen, to the fact that they have constituent parts and that they are productive, systematic, and compositional.

The classical approach in cognitive psychology purports to model this chain of description levels by introducing a lower description level whose states are defined by reference to a cognitive system that stores and processes certain syntactic formulae and adopts computational relations towards them.

Description level Classical.0: States are constituted by syntactic formulae and computational relations to these syntactic formulae. The structure of these states tends to coincide with that of Folk.1 states, except in the fact that the objects and properties of Classical.0 states are individuated only by their shape.

What a classical representational model would claim is that it is possible to establish a mapping from each state in Folk.1 onto a different state in Classical.0 by mapping the semantic properties of Folk.1 states onto syntactic properties. Positing description level Classical.0, together with the further claim about the possibility of mapping such 
states onto Folk.1 states, amounts to assuming, in Fodor's terms, the Language of Thought Hypothesis. ${ }^{10}$ That is, the idea that the content of our thoughts and the rules that govern their processing are explicitly encoded in our brain by means of syntactic formulae that mimic their content.

We can also define a description level Classical.2 where Classical.0 states will appear as objects and different sorts of properties are ascribed to them. ${ }^{11}$ Classical. 2 and Folk. 2 are closely connected because Classical.0 states surely possess constituent parts and are productive, systematic, and compositional.

One habitual objection to this model is that propositional attitudes in Folk.1 are individuated by reference to elements that go beyond the intrinsic properties of the organism to which they are ascribed, whereas classical models understand states in Classical.0 as autonomously or individualistically individuated.

I do not want to discuss this controversial issue now and, for the sake of argument, I shall feel content with the retort that Folk.1 can be revised in such a way that its states possess narrow contents, i.e., states whose contents can be individuated on the basis of the intrinsic properties of the organism. We will call this new description level Folk'.1. Description level Folk.2 should also be modified in the same direction whereby we would obtain Folk' $.2{ }^{12}$

To come to the objection we are interested in, we must recall that, as Fodor himself acknowledges, the main argument for the Language of Thought Hypothesis, and, in the end, for a classical approach to a representational conception of mind, has the structure of an affirmation of the consequent, or, to put it another way, the structure of an inference to the best explanation. In particular, Fodor claims that Classical.0 states are indispensable to account for the systematicity, productivity, and compositionality of our thoughts, and no alternative, better grounded story is available.

Nevertheless, once connectionist models of our cognitive capacities are developed, this inference to the best explanation cannot be applied so easily. For some people have argued that connectionist models may set the bases for such an alternative story. ${ }^{13}$ This is precisely the question I want to address in this paper.

Despite the variety of connectionist models, I will seek to point out a number of features that all of them share. ${ }^{14}$ Thus, we can say that the basic elements of a connectionist system are constituted by very 
simple elements called 'units'. These units are interconnected, setting up patterns of connectivity. Connections among units can have distinct strengths and weights, and they can be positive or negative, i.e., excitatory or inhibitory. Each unit that is on in the processing has an activation value that is a function of the global strength, either negative or positive, of the inputs that it receives and its bias, which is a value that the system associates with each unit. The influence that a given unit $\mathrm{A}$ has upon another unit $\mathrm{B}$ is a function of A's activation value and the weight and sign of the connection. Units are organized in networks, which, in turn, can have different sorts of architecture. Thus, networks may have a mere horizontal structure, but, in some models, they will also have a vertical or multi-layer organization. Networks can also be interconnected in various ways, and the whole system can be described as a network of unit networks.

A good way of illustrating how a connectionist system encodes and transforms representations is by viewing it as an interconnected set of constraint satisfaction networks. On this view, each input unit of the system is construed as representing a certain hypothesis about the presence of a given semantic, visual, acoustic, etc., feature. Input units will be on with a certain activation value when the feature that they represent is present, or off, otherwise.

Connections among units are to be interpreted as constraints among hypotheses. If unit $\mathrm{A}$ has a strong positive connection with unit $\mathrm{B}$, this means that for the system anything that has the feature represented by unit A is likely to have the feature represented by unit B, and inversely when the connections have a negative weight. The particular value of this weight determines the extent to which a given hypothesis imposes a constraint upon a second hypothesis.

Propositional contents - not just features - are not located in any particular unit, but their location is distributed across the network. Correspondingly, the same unit can participate in representing more than one propositional content and, relatedly, the same propositional content can be encoded in more than one network.

Once a connectivity pattern has been activated by a set of inputs, it gives rise to a pattern of activation that tends to settle, i.e., to relax to a solution. The system does not need to satisfy all constraints in order to settle into a solution: it tends to relax to the solution with the highest degree of constraint satisfaction, even if a certain amount of constraints are not fulfilled. ${ }^{15}$ 
One of the major virtues of connectionist systems is that they can learn as a result of training, whereby they do not need to be told directly in order to modify progressively their outputs as a result of the inputs that they receive. As one could expect, a connectionist system learns by modifying its patterns of connectivity in accordance with specific learning rules. ${ }^{16}$

To sum up, we could say that connectionist models define a new description level.

Description level Connectionist.0: States are connectivity patterns and activation patterns. The objects of these states are input units, hidden units, and output units; while their nonindividuating properties are excitatory and inhibitory connections with a specific weight, and activation values of units in each particular processing.

As in the case of classical models, we can also introduce a metalevel of description Connectionist. 2 where Connectionist. 0 states will appear as objects and different sorts of properties will be ascribed to them. ${ }^{17}$

The emergence of description level Connectionist.0 may represent a threat to a classical representational theory of mind for, at least, two reasons. One, if a systematic correlation between Folk'.1 and Connectionist.0, and Folk'.2 and Connectionist.2 states can be established, then description level Classical.0 may become theoretically superfluous. For we must recall that the most powerful argument for postulating Classical.0 states, that is for the Language of Thought Hypothesis, is that there was no alternative, better grounded explanation for commonsense psychological states; but, if the aforementioned correlation can be fixed, then connectionist models might represent such an alternative story. Two, there are reasons for doubting the possibility of establishing a systematic correlation between Connectionist. 0 and Classical. 0 states. If this is true, then one of these two state types will not be able to correlate systematically with Folk'.1 states, so that classical and connectionist models will appear as incompatible. Hence, the more plausible connectionist models appear, the less plausible classical models become.

Let us explore these two questions by, firstly, putting forward two lines of argumentation in defence of classical models and, secondly, seeking to dismantle such arguments and assess, as a conclusion, the threat that connectionist models pose to classical models. 


\section{TWO LINES OF DEFENCE FOR CLASSICAL MODELS}

I intend to examine two lines of defence for classical models against the threat of connectionism. As we shall see, the first line claims that connectionist models, as opposed to classical ones, are not at a cognitive level, but at an implementation level, so they will constitute at most one of the possible implementations that classical models may receive. On this view, classical and connectionist models will not be incompatible but complementary.

The second line of defence questions one basic assumption of connectionist models, namely, the possibility of establishing a systematic correlation between Connectionist. 0 and Folk'. 1 states. If this correlation were not possible, then connectionist models could hardly be viewed as a challenge to classical models insofar as we maintain our commitment to the scientific respectability of folk psychology. ${ }^{18}$

\subsection{Fodor and Pylyshyn: The Compatibility Thesis}

We find the first line of argumentation in Fodor and Pylyshyn (1988). To begin, we ought to see the notion of cognitive level that they have in mind:

Since Classicists and Connectionists are both Representationalists, for them any level at which states are taken to encode properties of the world counts as a cognitive level; and no other levels do....

It follows that if you want to make good the Connectionist theory as a theory of cognitive architecture you have to show that the processes which operate on the representational states are those which are specified by a Connectionist architecture.... It is, in particular, perfectly possible that nonrepresentational neurological states are interconnected in the ways described by Connectionist models but that the representational states themselves are not. (Fodor and Pylyshyn 1988, pp. 9-10)

Accordingly, a model is at a cognitive level only if the states that it posits encode contents and the ways they are processed are the same as those of representational states. Fodor and Pylyshyn think that productivity, systematicity, and compositionality are the most basic features of the ways representational states are processed, and this can be explained in virtue of the fact that representational states have constituent parts.

To put it another way, we could say that a description level $\mathrm{A}$ is at a cognitive level only if (a) its states can be systematically mapped onto 
Folk'.1 states and (b) its states have, the same structural properties as Folk'.1 states, i.e., a description level can be defined that postulates a type of state where A states appear as objects and some of their properties coincide with those properties that folk psychology attributes to Folk'.1 states. Fodor and Pylyshyn focus on this latter respect, and their basic argument is:

(1) Folk'.1 states are productive, systematic, and compositional. (From Folk'.2)

(2) Connectionist.0 states have parts but not constituent parts.

(3) States are productive, systematic, and compositional only if they have constituent parts.

(4) Connectionist.0 states are neither productive, systematic, nor compositional. (From (2) and (3))

(5) It is not possible to define a description level Connectionist.2 that postulates a type of state where Connectionist.0 states appear as objects and some of their properties coincide with the properties that folk psychology attributes to Folk'.1 states. (From (4))

(6) Connectionist.0 states are not at a cognitive level. (From (5) and the definition of cognitive level)

Most of Fodor and Pylyshyn's principal argument rests on the notion of constituent part that appears in premise (2). They borrow this idea from certain analyses of the structure of language and apply it to the way thoughts are connected. This strategy is not at all surprising because classical theories take, by and large, linguistic structures as the model for thought structures.

In particular, Fodor and Pylyshyn presuppose a combinatorial syntax and semantics based on the distinction between atomic and molecular components, so that molecular representations have other representations as their components that, in turn, can be molecular or atomic. The analysis stops as we come to the atomic level. The content of molecular representations is a function of the content and syntactic articulation of its atomic parts.

On this view we can introduce the idea of a 'constituent part' of a molecular representation as a part that has a content and that, in construction with other contentful parts, supplies the content of the representation. On this account, it is easy to see that Classical.0 states 
do have constituent parts; what Fodor and Pylyshyn urge is that connectionist states do not.

One could be misled by the fact that connectionist models put labels on units and on connectivity patterns, and argue that labels on units correspond to the atomic parts of a molecular representation, whereas labels on connectivity patterns correspond to molecular representations. Correspondingly, we might feel tempted to say that labels on units are the constituent parts of molecular representations, whereas the way in which units are interconnected stands for their syntactic construction. This is the illusion that Fodor and Pylyshyn want to dispel.

What they contend is that, although units and connectivity patterns may be labelled, these labels do not play any role in the processing. For units are causally connected, but not semantically or syntactically linked. In other words, the outcoming representation owes its content not to the structure of the labels we choose for the distinct units, but to causal interconnections among the units. Accordingly, units in connectionist models cannot be construed as constituent parts of complex representations, since the content of the latter is not a function of the label of the units plus their syntactic construction. ${ }^{19}$

Fodor and Pylyshyn introduce some further remarks to make plausible premises (1) and (3) of their basic argument, although no real argument is supplied to ground them. But if we decide to concede these two premises, we would have to acknowledge that connectionist models are not at a cognitive level and that they constitute at most one of the possible ways in which a classical model can be implemented. Consequently, classical and connectionist models will not be incompatible, but complementary.

\subsection{Ramsey, Stich, and Garon: A Conditional Claim}

Ramsey, Stich, and Garon ${ }^{20}$ provide an argument against the possibility of establishing a mapping from connectionist states onto mental contents, or, in other words, against the possibility of defining a systematic correlation between Connectionist. 0 and Folk' 11 states. This argument may be read in two ways, either as a conditional claim from connectionism to eliminativism or as a conditional claim from folk psychology against connectionism. ${ }^{21}$ If we choose to read this argument in the latter direction, it would constitute indirect support for classical models, for these models certainly are compatible with folk psychology. 
What Ramsey and his colleagues defend may be summarized in the following quotation:

The thesis we have been defending in this paper is that connectionist models of a certain sort are incompatible with the propositional modularity embedded in commonsense psychology. The connectionist models in question are those which are offered as models at the cognitive level, and in which the encoding of information is widely distributed and subsymbolic. In such models, we have argued, there are no discrete, semantically interpretable states that play a causal role in some cognitive episodes but not in others. Thus there is, in these models, nothing with which the propositional attitudes of commonsense psychology can plausibly be identified. If these models turn out to offer the best accounts of human belief and memory, we will be confronting an ontologically radical theory change - the sort of theory change that will sustain the conclusion that propositional attitudes, like caloric and phlogiston, do not exist. (Ramsey et al. 1990, p. 520)

The paper begins by distinguishing between ontologically conservative and ontologically radical theory change. In the first class of change, the new theory assumes fundamentally the same ontological commitments as the old one. An ontologically radical theory change, on the other hand, refers to a case where the new theory is so deeply and fundamentally different from the old one that it involves a revision of its basic ontological commitments. This is what happened when entities like phlogiston and caloric were abandoned. Similarly, Ramsey and his colleagues claim that, if connectionism proved to be our best cognitive theory, then it would involve an ontologically radical change. For the ontological commitments of folk psychology and connectionist models are so disparate that it is not possible to establish a systematic correlation between folk psychology and connectionist states.

On their view, common-sense psychology is fundamentally committed to the existence of propositional attitudes as states that: (1) are discrete, (2) are semantically interpretable, and (3) play a causal role in the production of other propositional attitudes and, in the end, in the production of behaviour. They ascribe the label of 'propositional modularity' to this set of ontological commitments and suggest that connectionist states cannot meet these requirements.

Propositional modularity is in some ways a shorthand for the properties that folk psychology ascribes to Folk'.1 states. Thus, the fact that propositional attitudes have semantic content and causal powers forms a part of how Folk'.1 states are defined, and the suggestion that propositional attitudes are discrete is closely linked to the idea that Folk'.1 
states are productive, systematic, compositional, and, therefore, have constituent parts.

The key idea in the argument is that propositional attitudes are functionally discrete states, namely, that the causal role played by each propositional attitude can be isolated from the causal role played by any other propositional attitude. This idea is illustrated by introducing a semantic model for memory, where it seems clear that: (a) a propositional attitude can be added to (or subtracted from) the memory network without altering any of the remainder; (b) propositional attitudes are projectible predicates, namely, predicates that can be used in nomological generalizations; ${ }^{22}$ and (c) one can distinguish between those propositional attitudes that are activated and those that are not.

The connectionist model for memory that Ramsey and his colleagues propose, which is meant to represent the basic features of most connectionist models, does not seem to meet any of these conditions. The model shows how sixteen propositions and their respective truth values could be encoded in a connectivity network A with sixteen input units, four hidden units, and one output unit. About this system we can truly say that it stores information about the truth value of these sixteen propositions, but we cannot specify which part of the network encodes each particular proposition. Thus, (a) if we wish to add a seventeenth proposition to our original network, we would need to rearrange the whole network. And (b), as there are so many possible connectionist networks that can store information about a proposition (think that the way in which it is stored depends, for example, on how other previous propositions have already been encoded), there is no projectible feature that could be isolated at a connectionist level.

One could reply that, even if we grant (a) and (b), this will not necessarily prove that we could not specify how the network encodes each particular proposition. But this encoding has important limitations, since it is relative to each particular network and, therefore, excludes any projectibility. Moreover, we cannot say that this specification will help us to single out a discrete functional role with respect to other propositional attitudes at any particular moment. In other words, the most we could establish is a token-token correlation between the functional role of a propositional attitude and a connectionist state. But this falls short of what was required, i.e., a systematic mapping from propositional attitudes onto connectionist states. 


\section{CONNECTIONIST THEORIES OF COGNITION}

I do not find the two arguments set out in the previous section very convincing. In fact, I intend to argue that Fodor and Pylyshyn's argument may be sound but proves too little, whereas the conditional claim rests upon assumptions that I would like to challenge. As a result, I will conclude that neither of the two arguments considered excludes the possibility of viewing connectionist models as forming a part of a representational theory of cognition that dispenses with the Language of Thought Hypothesis. Finally, I will run a pair of arguments against a realist interpretation of classical models on the basis of the strategies employed to dismantle the compatibility thesis and the conditional claim.

\subsection{Multi-level Cognition Theories}

Fodor and Pylyshyn claim that connectionist models are not at a cognitive level because their states do not have constituent parts. I would agree to this, but there are a couple of questions that I would like to ask: (a) Why should a cognitive theory define its basic description level at a cognitive level?; and (b) Do lower description levels not impose constraints upon higher description levels?

Question (a) points to the idea that cognitive theories must be concerned not only with the properties of the states at a cognitive level, but also with those of states at lower levels of description. In general, I would say that, within a representationalist approach to our cognitive capacities, ${ }^{23}$ a lower level of description can integrate into a cognition theory only if mental contents and their structural properties can be viewed as emerging from the lower-level state types and their structural properties. In other words, a lower level of description forms a part of a representational theory of cognition only if a systematic relation can be established between mental content types and their structural properties, on the one hand, and those lower-level state types and their structural properties, on the other.

I cannot see anything in Fodor and Pylyshyn's argument to prevent us from viewing connectionist states as the fundamental lower-level states of a representational theory of cognition, since nothing in their argument excludes the possibility of establishing the type-type relations 
that our notion of cognition theory requires. The only thing that their argument rules out is that connectionist states have constituent parts, but it does not supply any reason to reject the possibility of regarding the productivity, systematicity, and compositionality of mental contents as emerging from states that do not have constituent parts.

In fact, they seem to assume that such an emergence is possible. For they consider that connectionist models can implement classical models, and therefore they seem to grant that a systematic relation can be established from connectionist state types and their structural properties to mental contents and their structural properties.

We must, then, conclude that no principled reason has been supplied, for now, against the idea that connectionist models can be incorporated into a representational theory of cognition that involves description levels Connectionist.0, Folk'.1, Folk'.2, and Connectionist.2, as opposed to a representational theory of cognition developed from a classical point of view, which includes description levels Classical.0, Folk'.1, Folk'.2, and Classical.2. The reference to a neurological description level will be required in both cases if we want to account specifically for animal and human cognitive capacities. Let us call this new description level 'Neurological. -1 '.

Let us now consider a further consequence of this conclusion. Resuming question (2), we can show how what is true at lower levels of description imposes constraints on what can be defended at higher levels of description.

Thus, it is important to remember that the fundamental arguments for the Language of Thought Hypothesis have the structure of an inference to the best explanation. Then, if we show that a representational theory of cognition can dispense with Classical. 0 states because mental contents and their structural properties can be viewed as emerging from connectionist states, then we will have either to supply further evidence for the Language of Thought Hypothesis ${ }^{24}$ or to accept its superfluousness.

In fact, it can be argued that a connectionist theory of cognition can dispense with the Language of Thought Hypothesis on the basis of what Fodor and Pylyshyn have already granted. Thus, if we accept that classical states can be realized in a connectionist system, then we will have to accept that states with constituent parts can be instantiated in states without constituent parts. But, this being so, why couldn't mental contents be realized in a connectionist system without positing classical 
states?; in other words, why couldn't the constituent parts of mental contents and the systematicity, productivity, and compositionality that they generate be directly instantiated in the states of a connectionist system? I see no reason, in Fodor and Pylyshyn (1988), against an affirmative answer to this question.

To sum up, we can say that Fodor and Pylyshyn do not appear to have provided any reason to ground the need for the Language of Thought Hypothesis if we accept: (i) that connectionist systems can implement classical models and, therefore, mental contents; and (ii) that my notion of a representational theory of cognition constitutes an adequate characterization of the task that representational cognition theories are supposed to carry out. Accordingly, we can conclude that, even if classical and connectionist models are not necessarily incompatible, the emergence of the latter seems to undermine the best arguments for the Language of Thought Hypothesis, i.e., for the positing of Classical.0 states.

\subsection{A Holistic Constraint}

The conditional claim defended by Ramsey and his colleagues seems to affect the relation between connectionist models and folk psychology in a deeper way, since it implies that a systematic transition from Connectionist. 0 to Folk'.1 is not possible, in opposition to what a representationalist and connectionist theory of cognition would contend. Notice, however, that the conditional claim only backs an incompatibility thesis between connectionist theories and the classical picture of folk psychology; however, I am not convinced of the accuracy of this picture. In fact, it seems to have been shaped in accordance with the needs of classical models and not inversely.

There are some important elements of folk psychology that the classical picture seems to miss. For example, the strict causal discreteness of propositional attitudes is threatened by the presence of a holistic constraint in the explanations and predictions of actions that folk psychology supplies.

Suppose a subject $\mathrm{S}$ performs an action $\mathrm{A}$ that is explained by the belief B.1, although belief B.2 could also have led $\mathrm{S}$ to perform the same action. It surely makes sense to say that belief B.1, and not belief B.2, has played a causal role in the performance of A by S. The fact that we can distinguish those beliefs that have actually played a causal 
role from those that have not appears to support the classical picture. On the other hand, it would be intuitively odd to think that B.1 can explain A if it is not against an extensive background of skills, beliefs, and desires that $\mathrm{S}$ possesses. Moreover, we can say that the propositional attitudes that we can ascribe to $\mathrm{S}$ coincide with the propositional attitudes that enable us to explain and predict his actions. ${ }^{25}$ But if this is so, then it seems that each of S's beliefs cannot be fully individuated independently of the subject's cognitive background and, therefore, that their causal role is not entirely discrete. For if this background were significantly altered, the content of each belief truly ascribed to $\mathrm{S}$ and its corresponding causal role would vary, too. A parallel argument could be run for the predictions of actions.

Thus, we will obtain a different picture of the properties that from description level Folk'.2 we can truly ascribe to Folk'.1 states. We will still have propositional attitudes with a certain degree of causal discreteness but only against a background of skills and propositional attitudes. There is no need, however, to think of this background as entirely unstable and notably affected by every new input. So, we need not suppose that common-sense explanations and predictions of actions are strictly holistic, but neither can we assume that they are strictly discrete or modular.

It seems that connectionist systems conform to this restricted version of the holistic constraint, since the holistic character of Connectionist. 0 states is not incompatible with a certain degree of discreteness as long as they can be organized in distinct modules or networks with some excitatory and inhibitory connections between each other. This discreteness may have a horizontal structure, but it may also give rise to a multi-layer system. If we add to this moderate notion of modularity the fact that most new inputs can be stored with a smallish modification of our previous connectivity patterns, then it becomes possible to individuate Connectionist. 0 states across differences in background, as in the case of ordinary propositional attitudes.

Connectionist systems can also mimic the common-sense distinction between those representations that are operating at a given moment and those that are not, once we have individuated the specifications in connectivity patterns that encode each representation. Thus, we can say that a certain representation is operative in a computational processing when the specifications in connectivity patterns that encode it are 
activated across a given network of the system, otherwise we will say that this representation is just passively present in the system. The extent to which this distinction between operative and passive representations can be viewed as a clear-cut dichotomy will depend on the degree of discreteness allowed by each connectionist system. Thus, very often it would be more appropriate to speak of different degrees of operativity or passiveness. In any event, we could say, in our previous example, that belief B.1 caused S's action, and not belief B.2, inasmuch as the former was operative in the processing while the latter was not.

One could argue that what the argument of Ramsey and his colleagues excludes is precisely the possibility of individuating the activation pattern in a connectionist system that encodes a certain representation. Nevertheless, their argument is sound only if we assume that representational contents are strictly discrete. If, by contrast, we grant that the individuation of both Connectionist.0 and Folk'.1 states is subject to a version of the holistic constraint compatible with a certain degree of discreteness, then nothing in their argument prevents us from establishing this systematic correlation.

There are, however, some further problems in our way to establish a systematic correlation between Folk' ${ }^{\prime} .1$ and Connectionist. 0 states. I will briefly point out some of them.

To start, one could say that the fact that Connectionist.0 states can be individuated in a much more accurate and precise way than Folk'.1 states challenges the possibility of a strict systematic correlation. For, if a mapping between states of these two types could be at all established, it would involve such a remarkable degree of vagueness or coarseness that its explanatory relevance will diminish significantly.

In a slightly different direction, one could point out that, whereas it makes sense to produce a complete description of a system within a description level Connectionist.0, it is not so obvious that a complete description could be supplied within description level Folk' ${ }^{\prime}$.1. For our ordinary psychological explanations appear to be context-dependent, at least in the sense that our individuation of a subject's propositional attitudes is usually a function of rather specific pragmatic interests. We assume that these propositional attitudes are individuated against a background of skills and propositional attitudes that can be rendered partly explicit if required; but there seems to be no obvious way to yield a complete description of a subject's propositional attitudes. Nev- 
ertheless, if a correlation between Connectionist. 0 and Folk' 1 states is going to be possible, then such a complete description of Folk' 1 states would be required.

One way of escaping these two obstacles is claiming that a connectionist theory of cognition is only indirectly interested in such a mapping between Folk'.1 and Connectionist.0 states. For what is really needed is a systematic correlation between Connectionist. 0 states and a sort of representational state stipulated by a new description level, Representational.01, rather distant from description level Folk' ${ }^{\prime}$, but still representational and individualistic. Representational.01 states will also differ from Classical. 0 states because the former cannot be strictly syntactic and discrete, although they are committed to a new version of the Language of Thought Hypothesis, which, in opposition to the traditional one, is still in need of an initial clarification of its central features. ${ }^{26}$

In any event, it seems clear that the major current contribution of connectionism to the understanding of our cognitive capacities is not to have supplied correlations between connectionist and representational states, but to have shown how a physical system can naturally possess some of the most striking properties of our cognitive capacities. For example, soft-constraint satisfaction, spontaneous generalization, content-addressable memory, gradual-concept acquisition, graceful degradation, etc. All these features are in some way connected to the holistic constraint, but unfortunately I do not have room here to analyze how connectionist models could account for them. ${ }^{27}$ I want simply to suggest that, on my account, they will constitute properties of both Folk'.1 and Connectionist. 0 states that are ascribed from their respective metalevels of description. This fact suggests that a strong systematic correlation can be established between the properties truly attributed to the objects that compose Folk' .2 and Connectionist. 2 states.

\subsection{Against Classical Models}

One can use the arguments developed in the two previous subsections not only to defend connectionism but also to attack classical models. At this point, we can distinguish two claims:

Claim (1): Classical.0 states cannot be systematically correlated to Folk'.1 states. 
Claim (2): Even if Classical.0 states can be systematically correlated to Folk'.1 states, they do not meet the constraints imposed by Neurological. -1 states.

Although claim (2) is weaker than claim (1), I consider that the truth of the former would be enough to undermine classical models as a plausible theory of how human and animal cognition actually operates.

With respect to claim (1), we can say that the argument that Ramsey and his colleagues run from functional discreteness to the impossibility of mapping from mental representations onto connectionist states can now be inverted and transformed into an argument that runs from the holistic constraint to the impossibility of a systematic mapping between mental representations and formulae in the Language of Thought.

Thus, suppose we set up a mapping from the causal roles of the formulae F.1, ., F.n in mentalese onto the causal roles of mental contents Mc.1, .., Mc.n. Observe what happens if we enter a new formula F.n +1 into the network - the rest of the set remains unaltered given that formulae in mentalese are functionally discrete; but if a new mental content appears, Mc. $n+1$, many elements of the network have to be rearranged, so that we get a new set $\mathrm{Mc}^{\prime} .1, \mathrm{Mc}^{\prime} .2, \ldots, \mathrm{Mc} . \mathrm{i}, \ldots$, $\mathrm{Mc}^{\prime} . \mathrm{n}, \mathrm{Mc} . \mathrm{n}+1$. Thus, the old mapping is no longer strictly valid and a new mapping is required, and this will happen each time that a variation in mental contents is produced.

Of course, this is a very simplistic case because the holistic constraint admits of a certain degree of discreteness whereby not all mental representations need to be affected by the introduction of new contents and, on the other hand, some modules may have rather stable backgrounds. Taking into account these two factors, one can expect that classical models may be good at supplying a coarse mimicking for those cognitive capacities that are carried out within modules with rather stable backgrounds. But we can no longer expect a strict systematic correlation between Classical. 0 and Folk' ${ }^{\prime} .1$ states.

Regarding claim (2), I would like to say that, even though classical models could supply mechanisms to mimic strictly all our cognitive capacities, they could hardly be construed as realistic models about the way our mind operates. For at a lower level, namely at a neurological level, our knowledge imposes some constraints upon what can be true at a cognitive level. ${ }^{28}$

To illustrate this point, let us briefly examine what is implicated in 
our capacity to perform multi-constraint tasks like preparing breakfast or playing soccer, where many variable features of the environment need to be taken into consideration at the same time. We are generally good at this sort of task. Our way of performing such tasks displays a number of properties that have been described as graceful degradation, tolerance to damage and noise, etc. What all these properties seem to have in common is that we are able to maintain the standard of our performance despite failures in the satisfaction of some constraints that ought to be met or, in any event, that degradation in our performance tends to be gradual.

This situation has been explained by the fact that the constraints that are involved in such tasks are not hard, but soft. ${ }^{29}$ I should say that a constraint is hard if its violation collapses the successful performance of the task, whereas a constraint is soft when it integrates a set of constraints, any one of which can be violated without collapsing the successful performance of the task. Of course, the increase in the number of soft constraints that are infringed on will reduce the accuracy of the performance leading to a process of graceful degradation.

It seems clear that connectionist models will naturally simulate softconstraint satisfaction, since, as we pointed out in Section 1.2, connectionist systems tend to settle into the solution with the highest degree of constraint satisfaction, even if not all constraints are satisfied. In fact, the softness of connectionist constraints represents one of the most appealing features of connectionist models.

The question now is whether classical models can simulate softconstraint satisfaction within the boundaries of our neurological structure. It seems clear that classical models can only produce explicit, hard rules, since Classical. 0 states are constituted by syntactic formulae and, therefore, all rules need to be stated syntactically. Syntactic objects are composed of discrete constituent parts. The question about whether two syntactic objects are identical is an all-or-nothing matter: it depends on whether all their constituent parts coincide. Similarly, the question about whether a certain rule applies to a syntactic formula will also be an all-or-nothing matter: it will depend on whether the latter has exactly the constituent parts specified by the former. The point is that if one of the constraints indicated by the rules is not satisfied, then the rule will not apply. Of course, a rule can include indications about possible exceptions, but, in any case, each possible exception needs to be explicitly stated. In other words, we can say that any attempt to simulate 
soft-constraint performances by classical models will require the introduction of further explicit, hard rules that specify how every exception will affect the development of the task.

We must remember, however, that the number of features of the environment that may be relevant to a multi-constraint task is indefinitely large and, therefore, that the number of possible exceptions or variations in the rule will also be indefinitely large. Hence, we can easily see that any attempt to model soft-constraint performances by means of hard constraints, as classical models seek to do, would lead to a sort of computational explosion. For, given that we are dealing with sequential processing, we would need to increase dramatically the speed and number of units implicated in the processing, not only to be able to perform the task at all, but also in order to perform it in real time.

All this suggests that classical models may be neurologically implausible because they structure their data in a way quite different from the way our brain operates. Rumelhart and McClelland (1986b) point out, for instance, that neurons are much more interconnected than classical units, and that learning in our brain seems to be associated with variations in neuronic interconnections rather than with alterations of the units themselves. Nevertheless, what seems to discard radically classical models as a realist model for our cognitive capacities is the fact that their modelling of our performance of multi-constraint tasks would go beyond the boundaries, established by Feldman and Ballard (1982), of the speed of neuronic processing. They argue that a plausible neurological model ought not to take more than one-hundred serial steps per second, but any classical algorithm designed to simulate any of the multi-constraint tasks we are quite good at would certainly go far beyond this upper bound. ${ }^{30}$

\section{CONCLUSIONS}

In this paper, I have examined a compatibility thesis and a conditional claim on the way classical and connectionist models relate. With respect to the compatibility thesis, I have argued that, even if classical and connectionist models are not necessarily incompatible, the emergence of the latter seems to undermine the inference to the best explanation upon which the Language of Thought Hypothesis, and therefore the positing of Classical.0 states, appears to rest. For an inference of this 
nature only holds if no alternative, better grounded explanation is available, and I have been arguing that nothing in Fodor and Pylyshyn's argument conflicts with the possibility that connectionist models integrate into a representational theory of cognition that, apart from being backed by stronger evidence, accounts for the structural properties of the mental representation without postulating the Language of Thought.

I have attacked the conditional claim presented by Ramsey and his colleagues by pointing to the presence of a moderate holistic constraint in the explanations and predictions of actions that folk psychology supplies. Thus, I have sought to show not only that connectionist models are in principle compatible with this moderate holistic constraint but also that classical models will have trouble meeting this constraint, since an inverted version of the conditional claim could be addressed against them.

Moreover, I have argued that classical models could hardly be construed as a realist approach to the way our mind operates. For it can be argued, in the context of a multi-level cognition theory, that classical models can hardly satisfy the constraints that our knowledge at a neurological level imposes upon what can be true at a cognitive level.

Accordingly, we are in a position to conclude that neither of the two lines of argumentation considered excludes the possibility of viewing connectionist models as contributing to a multi-level theory of cognition that, in spite of dispensing with the neurologically implausible postulation of the Language of Thought, is able to include a notion of mental representation continuous with the basic features of our folk psychology. ${ }^{31}$

\section{NOTES}

${ }^{1}$ Of course, both classical and connectionist models have also been used to support eliminativist stances with respect to folk psychology. In fact, what I seek to do, in this paper, is to assess whether classical and/or connectionist models can account, at least in principle, for folk psychology. An affirmative response would back intentional realism (cf. Fodor (1987, Chapter 1 and Appendix) for classical models, and Smolensky (1988) for connectionist models), whereas a negative answer would advise an eliminativist position (cf. Stich (1983) for classical models, and Ramsey et al. (1990) for connectionist models).

${ }^{2}$ This hypothesis was established in Fodor (1975). For a more recent vindication of this hypothesis, see Fodor (1987), Fodor and Pylyshyn (1988), Fodor and McLaughlin (1990). 
${ }^{3}$ In Ramsey et al. (1990).

4 In this context, I understand that the term 'property' concerns not only properties of single objects, but also relations among objects. I should also say that each description level will certainly supply more or less flexible criteria for distinguishing between the individuating and nonindividuating properties of objects.

5 In this paper I take the notion of systematic correlation as primitive, and I use 'mapping' as a synonym.

${ }^{6}$ As Fodor puts it, an intentional realist will assume: (i) "[t]hat there are beliefs and desires and that there is a matter of fact about their intentionai contents"; (ii) "[i]t may be that believing and desiring will prove to be states of the brain"; and (iii) "[t]hat beliefs and desires have causal roles and that overt behavior is typically the effect of complex interactions among these mental causes" (Fodor 1987, p. 135).

7 At this point I am following Fodor and Pylyshyn (1988).

${ }^{8}$ Of course, it is always possible to distinguish further description levels within folk psychology, but I only mean to specify those that are directly relevant to our discussion about connectionism.

${ }^{9}$ In order to account for these properties of propositional attitudes, we ought to introduce an intermediate description level where propositional attitudes will no longer be under consideration, but will be the distinct structures of these propositional attitudes. These structures will be determined by the analysis of the syntactic and semantic relations among propositional attitudes. An easy way to produce a general characterization of these states is reading as variables what in Folk.1 states appear as constants and eliminating the reference to time.

This intermediate description level will not be relevant, however, to my analysis of the debate between classical and connectionist models.

10 Cf. note 2.

11 Of course, both classical and connectionist models will also take into consideration that any account of human cognitive capacities must be neurologically instantiated, although, as we shall see, they stress this point quite differently.

${ }^{12}$ For the classical case against the notion of narrow content, cf. Putnam (1975) and Burge (1979). For a classical defence of this notion, cf. Fodor $(1975,1980)$ and Field (1978). Interesting discussions of this topic can be found in Woodfield (1982) and Pettit and McDowell (1986). For more recent criticism of narrow content, see Putnam (1988, Chapters 1-3) and an interesting revision of the concept in Fodor (1987, Chapter 2; 1991). 13 For indications in this direction, cf. Rumelhart and McClelland (1986b), Smolensky (1988), Clark (1989a), Horgan and Tienson (1989), and Hanson and Burr (1990).

14 A more detailed characterization of the general features of connectionist models can be found in Rumelhart et al. (1986), Smolensky (1988), and Hanson and Burr (1990). For a taxonomy of the different connectionist models, cf. Rumelhart et al. (1986, pp. 54-72) and Hanson and Burr (1990, pp. 472-76).

15 As we shall see later, this sort of constraint is usually characterized as soft, in contrast to hard constraints, that is, those whose violation entirely collapses the processing.

I want to stress, in any event, that our illustration of how representations are encoded in a connectionist system is, of course, extremely simplistic in many respects; and yet I find it useful because, in my view, it captures some of the basic ideas that guide the elaboration of actual, more sophisticated connectionist models. 
${ }^{16}$ A number of different learning rules have been proposed for connectionist systems. One of the first, and one which underlics many others, is due to Hebb:

When an axon of cell A is near enough to excite a cell B and repeatedly or persistently takes part in firing it, some growth process or metabolic change takes place in one or both cells, such that A's efficiency, as one of the cells firing B, is increased. (Hebb 1949 , p. 62)

Accordingly, we can say that when two units A and B of the same network are simultaneously excited, the connection strength increases. Inversely, when two units of the same network tend not to be simultaneously excited, then the connection is modified in an inhibitory direction. An interesting aspect of this learning procedure is that it is localized in the connections between units and it does not require overall supervision.

${ }^{17}$ Cf. Clark (1989a, pp. 188-96) for an interesting presentation of the different levels of description that may be implicated in connectionist models.

${ }_{18}$ This argument was originally proposed in Ramsey et al. (1990) to support a conditional claim from connectionism to eliminativism, but it can be transformed into a conditional claim from representationalism to classical models and against connectionism.

19 Thus, Fodor and Pylyshyn remark:

Strictly speaking, labels play no role at all in determining the operation of a connectionist machine; in particular the operation of the machine is unaffected by the syntactic and semantic relations that hold among the expressions that are used as labels. To put this another way, the node labels in a connectionist machine are not part of the causal structure of the machine.... Whereas, by contrast, the state transitions of classical machines are causally determined by the structure - including the constituent structure - of the symbol arrays that the machine transforms. (Fodor and Pylyshyn 1988, p. 17)

${ }^{20}$ Cf. Ramsey et al. (1990).

${ }^{21}$ In other words, one may want to use this argument to challenge the scientific respectability of common-sense psychology insofar as their states cannot find a scientific correlate, were connectionism to settle as the most satisfactory scientific approach to cognitive capacities. Or one might feel inclined to argue that what the gap proves is that connectionist theories cannot account for our cognitive capacities. The option between these two opposite readings will depend on the stance that each one wants to adopt with respect to the more generic issue of whether we can make sense of the idea of cognitive capacity without ascribing content to cognitive states. I do not intend to discuss this generic issue in the present text.

I would like to point out, however, that the fact that some people could adopt an eliminativist stance in regard to folk psychology would not necessarily rule out the possibility of elaborating; as a subfield of scientific psychology, an account of why the folk think about mental states the way they do (cf. Goldman 1993, p. 15).

${ }^{22}$ We can introduce generalizations like the following: if $X$ was told that $p, X$ will normally remember that he has been told that $\mathrm{p}$. These sorts of generalizations work no matter how different the psychological features of some given subjects might be from other points of view.

${ }^{23}$ At this point, I confine myself to a representationalist approach because Fodor and Pylyshyn are also committed to this kind of approach. 
${ }^{24}$ Of course, one expects the evidence for connectionist models to be stronger than a mere inference to the best explanation.

${ }^{25}$ Cf., for instance, Dennett (1971).

${ }^{26}$ Further suggestions in this direction are in Clark (1989b).

${ }^{27}$ One can find suggestive characterizations of how connectionist models could in principle account for these features of our cognitive capacities in McClelland et al. (1986, Clark (1989a, Chapters 5, 6), Horgan and Tienson (1989, pp. 50-54), and Hanson and Burr (1990).

${ }^{28}$ Of course, this claim becomes entirely intelligible in the context of what I have characterized as a multi-level cognition theory.

${ }^{29}$ For an interesting analysis of the distinction between soft and hard constraints, cf. Horgan and Tienson (1986).

${ }^{30}$ For an analysis of this point developed as a criticism of Fodor and Pylyshyn (1988), see Chater and Oaksford (1990). In Clark (1989a, Chapter 4), we find suggestions about the implausibility of classical models from the point of view of evolution.

${ }^{31}$ This paper has benefited from comments by Kathleen Wilkes, Terence Horgan, Stephen Mills, Michael Devitt, Ana Estela, Jerry Fodor, Josep Luis Prades, Carlos Moya, Andy Clark, Martin Davies, Stephen Stich and Tobies Grimaltos. I am particularly grateful to Gerard O'Brien for our discussion about connectionism. I am also obliged to two anonymous referees of Synthese for their helpful suggestions. Finally, I should also say that this paper forms part of a research project that I developed at Oxford University during the academic year 1988-89, thanks to a grant awarded by the Valencian Council of Culture, Education and Science.

\section{REFERENCES}

Burge, T.: 1979, 'Individualism and the Mental', in P. A. French et al. (eds.), Midwest Studies in Philosophy 4, 73-121.

Chater, N. and M. Oaksford: 1990, 'Autonomy, Implementation and Cognitive Architecture: A Reply to Fodor and Pylyshyn', Cognition 34, 93-107.

Clark, A.: 1989a, Microcognition, Philosophy, Cognitive Science. and Parallel Distributed Processing, MIT Press, Cambridge, Massachusetts.

Clark, A.: 1989b, 'Connectionism, Non-conceptual Content and Representational Redescription', University of Sussex, Brighton, Serial no. CSRP 143.

Dennett, D.: 1971, 'Intentional Systems', Journal of Philosophy 8, 87-106.

Feldman, J. and D. Ballard: 1982, 'Connectionist Models and their Properties', Cognitive Science 6, 205-54.

Field, H.: 1978, 'Mental Representation', Erkenntnis 13, 9-61.

Fodor, J.: 1975, The Language of Thought, Harvester Press, Hassocks, Sussex.

Fodor, J.: 1987, Psychosemantics, MIT Press, Cambridge, Massachusetts.

Fodor, J.: 1980, 'Methodological Solipsism Considered as a Research Strategy in Cognitive Psychology', Behavioral and Brain Sciences 3, 63-110.

Fodor, J.: 1991, 'A Modal Argument For Narrow Content', The Journal of Philosophy 83, 5-26.

Fodor, J. and B. McLaughlin: 1990, 'Connectionism and the Problem of Systematicity; Why Smolensky's Solution Doesn't Work', Cognition 35, 183-204. 
Fodor, J. and Z. W. Pylyshyn: 1988, 'Connectionism and Cognitive Architecture: A Critical Analysis', Cognition 28, 3-71.

Goldman, A. I.: 1993, 'The Psychology of Folk Psychology', Behavioral and Brain Sciences 16, 15-28.

Hanson, S. and D. J. Burr: 1990, 'What Connectionist Models Learn: Learning and Representation in Connectionist Networks', Behavioral and Brain Sciences 13, 471 89.

Hebb, D. O.: 1949, The Organization of Behavior, John Wiley, New York.

Horgan, T. and J. Tienson: 1989, 'Representations without Rules', Philosophical Topics 17, $147-74$.

McClelland, J. L., D. E. Rumelhart, and G. E. Hinton: 1986, 'The Appeal of Parallel Distributed Processing', in Rumelhart and McClelland (1986a), pp. 3-44.

Pettit, P. and J. McDowell (eds.): 1986, Subject, Thought and Context, Oxford University Press, Oxford.

Putnam, H.: 1975, 'The Meaning and 'Meaning', in H. Putnam, Philosophical Papers, Vol. 2: Mind, Language and Reality, Cambridge University Press, Cambridge, pp. 215-71.

Putnam, H.: 1988, Representation and Reality, MIT Press, Cambridge, Massachusetts.

Ramsey, W., S. Stich, and J. Garon: 1990, 'Connectionism, Eliminativism, and the Future of Folk Psychology', in J. Tomberlin (ed.), Philosophical Perspectives, Vol. 4, Ridgeview, Atascadero, California, pp. 499-533.

Rumelhart, D. E., G. E. Hinton, and J. L. McClelland: 1986, 'A General Framework for Parallel Distributed Processing', in Rumelhart and McClelland (1986a), pp. 45-76. Rumelhart, D. E. and J. L. McClelland (eds.): 1986a, Parallel Distributed Processing Explorations in the Microstructure of Cognition, 2 vols., MIT Press, Cambridge, Massachusetts.

Rumelhart, D. E. and J. L. McClelland: 1986b, 'PDP Models and General Issues in Cognitive Science', in Rumelhart and McClelland (1986a), pp. 110-46.

Smolensky, P.: 1988, 'On the Proper Treatment of Connectionism', Behavioral and Brain Sciences 11, 1-74.

Woodfield, A. (ed.): 1982, Thought and Object. Essays on Intentionality, Clarendon Press, Oxford.

Departament de Metafísica i Teoria del Coneixement

Universitat de València

Av. Blasco Ibáñez, 21

46010 València

Spain 